\title{
Contextual Non-Inflammation Self-Promotion and Subsequent Origin as Oncogenesis
}

\author{
Lawrence M Agius* \\ Department of pathology, University of Malta Medical School, Europe
}

Submission: May 30, 2018; Published: June 25, 2018

"Correspondence Address: Lawrence M Agius, Department of pathology, Mater Dei Hospital, TAL-QROQQ, University of Malta Medical School, Msida, Malta Europe, Tel: 356-21451752; Email: lawrence.agius@um.edu.mt

\begin{abstract}
Performance identity is a proportional index of the cell injury that repeatedly characterizes and re-characterizes the dimensions of nonresolution of death pathways as systems of non-inflammation. The identity profiles for further change are by intrinsic nature a system profile that sharply demarcates adjacent healthy cells within the productive milieu of transforming homeostatic control mechanisms. The further significant emergence for potential change is centered paradoxically on the evolving dimensions of apoptosis inhibitors that clearly regulate the profile identity of progression systems in terms of non-resolution and as integral component for further transformation of cell components in carcinogenesis
\end{abstract}

\section{Introduction}

The essential contextual non-inflammatory or even potential anti-inflammatory nature of apoptosis is regulatory sub-stratum for the evolutionarily conserved dimensions for systems of intra-cellular and extrinsic operative fields in programmed cell death in cells currently undergoing malignant transformation. Calprotectin is necessary for the progression of non-inflammation driven hepatocarcinoma and may constitute a therapeutic target for the management of hepatic carcinoma formed in non-cirrhotic livers [1]. No significant correlation was found between chronic prostatic inflammation and prostatic carcinoma; inflammation was of higher score in benign prostatic hyperplasia and also total prostatic specific antigen levels were lower in the inflammation group [2]. Such scenario includes the cascade events of apoptosis in a manner derived from systems of activation and inhibition that evolve in terms particularly of permeation of the outer mitochondrial membrane and thus indicates a significant role for ionic imbalance in apoptosis activation.

\section{Significance of Non-inflammation}

The proportional nature included within the systems of cell death involves the dimensional compass of whole cell evolution in terms also of the glucocorticoid receptor. Apoptosis mediated by Fas antigen may be important in the pathogenesis of chronic hepatitis B [3]. Inflammation involves several wellknown secreted proteins with multiple functions in different cellular locations, with especially chronic inflammation that can last for months or years [4]. The species formulation within system pathways initiates the global phenomenon of potential malignant cell that is parallel to and antagonistically inhibitory to the emergence of pro-apoptosis as well-defined by primary thymocytes and other lymphoid-type cells when exposed to glucocorticoids.

Ganglioside sialidases in mammals have been implicated in the regulation of cell differentiation, cell growth and cell adhesion and motility depending on particular attributes [5]. The provocative inclusion of cell-death initiation evolves within encompassed formulas that include the terminal cascades common to both the intrinsic and extrinsic apoptosis pathways. The systemic immune-inflammation index may serve as a useful biomarker to predict recurrence in patients with advanced nonsmall-cell lung cancer [6]. The derivative inclusions of various forms of programmed cell death involve the caspases as primarily pro-apoptotic mechanisms that transform injured and stressed cells in the form of subsequent deliberation as shrunken and small retracted cell forms within otherwise healthy dimensions of tissue and organ homeostasis.

\section{Proportional constitution}

Contextual non-inflammation is a negative but also a dynamically evolving form of injury to cells in its own right that further defines the boundaries for cell derivation and cell fate within communities of cells that re-define the cascade events for both apoptosis and non-apoptosis in malignant transformation. 
Extracellular vesicles mediate signaling that directs the activities of surrounding cells and act as a positive feedback loop in cancer progression [7]. The incremental augmentation in caspase activation is reactive formula for a series of over-lapping and cooperative factors in inducing such non-inflammation. Anaesthesia type may influence oxidative stress, inflammation, molecular regulators and triple-negative breast cancer outcome [8]. The performance for injurious dimensions in cooperative dimension allows a permissive micro-environment in productive resolution of the cell injury that transcends aspects of cell transformation in oncogenesis. Evidence is emerging of cell-death-independent; non-apoptotic functions of caspases and may prove potential targets as a therapeutic strategy [9]. Expression of the anti-apoptotic mutations does not confer an advantage for tumor cells either in the short-term or long-term tumor formation. Cytotoxic CD8+ T cells are the main executors of transformed and cancer cells during cancer immunotherapy [10].

Human papillomavirus infection accounts in part to an increased incidence of squamous cell carcinoma of the head and neck but it is not known whether HPV influences fatigue or inflammation [11]. Profile constitution and reconstitution is a central referential index for cooperative activation in terms of a series of attempted resolution that is also para-homeostatic in principle. Dubbed as the new "aspirin" of current times, angiogenic dysregulation by metformin provides exciting and promising alternate strategy [12]. The divergent nature for such non-inflammatory conditions is milieu-specific within systems for cooperative reference points in subsequent tissue/ organ homeostasis. CD177 can be used to represent infiltration of neutrophils, which may be of potential prognostic value in pancreatic ductal adenocarcinoma [13]. The proper dimensions for resolution are subject to a cascade series of activation pathways that contrast with the loss of plasma membrane and mitochondrial membrane potential subsequent to ionic channel injury.

\section{Dynamics of Operability}

The dynamic profiles of operability of non-inflammation include the proportional cascade events in apoptosis that transcend proper dimensions for cell injury in a manner conclusively productive of cell loss within the equilibrating balance for homeostatic control.

In the referential cooperative series of transactions, the system profiles for potentially further injury to the cell resolve within the encompassed activities for further change as earmarked by a series of interventional transformations in oncogenesis. Proline-rich protein tyrosine kinase 2 stimulates multiple oncogenic signaling pathways that include Wnt/betacatenin, PI3K/Akt, MAPK/ERK, and TGF-beta/EGFR/VEGF; it enhances carcinogenesis, migration, invasion, epithelialmesenchymal transition and metastasis [14]. Performance attributes of non-inflammation are prime candidates for further cellular transformation within the system identities of homeostatic control of tissues and organs. It is further to such considerations that provocative activations more clearly outline the production of cellular phenotypes as dictated by performance profiles that counter-act the various potential resolution pathways as further profiled by mitochondrial and DNA injury. Chloroquine and hydroxychloroquine have antitumor properties due to strong anti-proliferative, antimutagenic, and inhibitory autophagy capacities in infectious, immune, neoplastic and neurologic disease [15]. Cationic nanoparticles can be used as effective in situ vaccine platform with both tumor destruction and immune activation [16].

\section{Central Cooperative Events}

Emergence profiles thus include the systems of central cooperative factors such as the inhibitors of apoptosis family of proteins (IAPs). It is within the essential controlling dimensions of IAPs that the significance of non-inflammation emerges as potentiality for further change in cellular profile as dictated by sub-components of that same cell host. The different inflammasomes and inflammatory cytokines and chemokine play contrasting roles in carcinogenesis and progression that may be related to release of pathogen-associated molecular patterns and damage-associated molecular patterns [17]. The proportionalities for change are direct profiles for an injury that is specifically non-inflammatory. Mutant K-RAS has both tumorpromoting and -suppressing functions, and the RASSF family of tumor suppressors may act as RAS apoptosis and senescence effectors [18]. The productive factor activation is in direct contra-distinction to the manner of injury to the cellular DNA on the one hand, and of mitochondrial dimensions of membrane potential loss and of prior permeabilization and poration of the membranes. The acquisition of resistance to endo- or xeno-biotic toxicity is critical for cancers that develop in toxic microenvironment [19].

\section{System Non-Resolution}

Resolution of apoptotic cascades is dimensionally incorporated within the boundary membranes of the mitochondria in the first instance that further re-defines the non-inflammatory nature of protease dysfunction in apoptosis progression. The gut microbiota may be modified to positively impact levels of systemic pro-inflammatory cytokines [20]. It is clearly in terms of re-defined cascade events that inhibitors of apoptosis emerge as the central cooperative system in noninflammation progression as well-illustrated by the shrinkage and condensation of the cell and of sub-components such as mitochondria and DNA.

The nature of non-inflammation hence is proposed dimension for a markedly active and reactive series of compound phenomena that re-characterize the system profiles for homeostatic organization and contribution to the pathways 


\section{Cancer Therapy \& Oncology International Journal}

of cascade nature. A sustained high neutrophil-to-lymphocyte ratio from baseline to mid-neoadjuvant chemotherapy is an independent prognostic factor in patients with muscle-invasive bladder cancer [21].

Substantial progression in apoptotic cascades resolve within the profile dimensions of nearby healthy cells within further confirmatory outlines for subsequent change as transformational carcinogenesis. It is believed that the proportional dimensions of non-inflammation clearly dictate the progression of cell-death pathways that specifically impact the resolving or non-resolving progression of injury to mitochondria and cellular DNA.

\section{Concluding Remarks}

The central machinery in malignant oncogenesis is referential system pathway as constituted by non-inflammation in tissues and organs as further dictated by the nature of cascade events in apoptosis precipitation. In like manner, the performance dynamics of non-resolution originate as conditioning and re-conditioning of system pathways of activation, and also potential loss of membranes and of mitochondria, and as further propagated by DNA shrinkage and condensation. The system profile injury to the cellular subcomponents is thus a para-homeostatic series of events that incorporate profile loss of characterization of sub-component biology. The systems of inhibition of apoptosis are hence the determinant profiles for non-inflammation that receive the characterized dimensions for potential further change that promotes carcinogenesis.

Incremental dimensions for such change are ideally a transforming influence in the cascade pathways that precipitate either apoptosis or non-apoptosis of individual cells that recharacterize injury in terms of subsequent events in programmed cell death.

\section{References}

1. De Ponti A, Wiechert L, Schneller D, Pusterla T, Longerich T, et al. (2015) A pro-tumorigenic function of S100A8/A9 in carcinogeninduced hepatocellular carcinoma. Cancer Lett 369(2): 396-404.

2. Engelhardt PF, Brustmann H, Seklehner S, Riede CR (2013) Chronic asymptomatic inflammation of the prostate type IV and carcinoma of the prostate: is there a correlation? Scand J Urol 47(3): 230-235.

3. Zhu K, Zhang L, Wang H, Wang S, Wang L, et al. (1997) Primary study on Fas-mediated apoptosis in the liver tissue of patients with chronic hepatitis B. Zhonghua Shi Yan He Lin Chuang Bing Du Xue Za Zhi 11(4): 336-369.

4. Yoon JH, Ryu J, Baek SJ (2018) Moonlighting activity of secreted inflammation-regulatory proteins. Yonsei Med J 59(4): 463-469.

5. Miyagi T, Takahashi K, Yamamoto K, Shiozaki K, Yamaguchi K (2018) Biological and pathological roles of ganglioside sialidases" Prog Mol Biol Transl Sci 156: 121-150.
6. Guo D, Zhang J, Jing W, Liu J, Zhu H, et al. (2018) Prognostic value of systemic immune-inflammation index in patients with advanced nonsmall-cell lung cancer. Future Oncol, UK.

7. Litwinska Z, Kuczkowska K, Machalinski B (2018) Extracellular vesicles in hematological malignancies. Leuk Lymphoma 10: 1-8.

8. Lukoseviciene V, Tikuisis R, Dulskas A, Miliauskas P, Ostapenko V (2018) Surgery for triple-negative breast cancer-does the type of anaesthesia have an influence on oxidative stress, inflammation, molecular regulators, and outcomes of disease? J BUON 23(2): 290295.

9. Wilson CH, Kumar S (2018) Caspases in metabolic disease and their therapeutic potential" Cell Death Differ.

10. Jaime-Sanchez P, Catalan E, Uranga-Murillo I, Aguilo N, Santiago L, et al. (2018) Antigen-specific primed cytotoxic T cells eliminate tumor cells in vivo and prevent tumor development, regardless of the presence of anti-apoptotic mutations conferring drug resistance. Cell Death Differ.

11. Xiao C, Beitler JJ, Higgins KA, Glazer T, Huynh LK, et al. (2018) Associations among human papillomavirus, inflammation, and fatigue in patients with head and neck cancer. Cancer.

12. Soobryan N, Murugesan S, Pandiyan A, Moodley J, Makckraj I, et al. (2018) Angiogenic Dysregulation in pregnancy-related hypertension-a role for metformin. Reprod Sci 1: 1933719118773484.

13. Wang Y, Fang T, Huang L, Wang H, Zhang L, et al. (2018) Neutrophils infiltrating pancreatic ductal adenocarcinoma indicate higher malignancy and worse prognosis. Biochem Biophys Res Commun 501(1): 313-319.

14. Zhu X, Bao Y, Guo Y, Yang W (2018) Proline-rich protein tyrosine kinase 2 in inflammation and cancer" Cancers (Basel) 10(5).

15. Plantone D, Koudriavtseva T (2018) Current and future use of chloroquine and hydroxychloroquine in infectious, immune, neoplastic, and neurological diseases: a mini-review. Clin Drug Investing.

16. An M, Yu C, Xi J, Reyes J, Mao G, et al. (2018) Induction of necrotic cell death and activation of STING in the tumor microenvironment via cationic silica nanoparticles leading to enhanced antitumor immunity. Nanoscale 10: 9311-9319.

17. He Q Fu Y, Tian D, Yan W (2018) The contrasting roles of inflammasomes in cancer. Am J Cancer Res 8(4): 566-583.

18. Schmidt ML, Hobbing KR, Donninger H, Clark GJ (2018) RASSF1A deficiency enhances RAS-driven lung tumorigenesis. Cancer Res 78(10): 2614-2623.

19. Collino A, Termanini A, Nicoli P, Disferia G, Polletti S, et al. (2018) Sustained activation of detoxification pathways promotes liver carcinogenesis in response to chronic bile acid-mediated damage" PLoS Genet 2018: 14(5).

20. Spychala MS, Venna VR, Jandzinski M, Doran SJ, Durgan DJ, et al. (2018) Age-related changes in the gut microbiota influence systemic inflammation and stroke outcome. Ann Neural.

21. Kaiser J, Li H, North SA, Leibowitz-Amit R, Seah JA, et al. (2018) The prognostic role of the change in neutrophil-to-lymphocyte ratio during neoadjuvant chemotherapy in patients with muscle-invasive bladder cancer: a retrospective, multi-institutional study. Bladder Cancer 4(2): 185-194. 


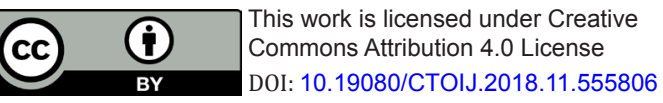

Your next submission with Juniper Publishers
will reach you the below assets
- Quality Editorial service
- Swift Peer Review
- Reprints availability
- E-prints Service
- Manuscript Podcast for convenient understanding
- Global attainment for your research
- Manuscript accessibility in different formats
( Pdf, E-pub, Full Text, Audio)
- Unceasing customer service
Track the below URL for one-step submission
https://juniperpublishers.com/online-submission.php

